\title{
Durational variability as a marker of prosodic structure in Indian English(es)
}

\author{
Elinor Payne ${ }^{1}$, Olga Maxwell ${ }^{2}$ \\ ${ }^{1}$ University of Oxford, UK \\ ${ }^{2}$ University of Melbourne, Australia \\ elinor.payne@phon.ox.ac.uk, omaxwell@unimelb.edu.au
}

\begin{abstract}
This paper presents an analysis of systematic durational variability as a marker of prosodic structure in the speech of university educated Indian English speakers from four L1 backgrounds (Hindi, Bengali, Tamil and Telugu), with the aim of investigating the degree of uniformity in Indian English prosody. The results reveal a complex picture, with some evidence for L1 influence, alongside possible convergence factors, both in the direction of native English features and in the direction of pan-Indian features. We discuss the implications of these findings with regard to the status and putative homogeneity of Indian English.
\end{abstract}

Index Terms: duration, variability, prosodic structure, Indian English

\section{Introduction}

'Indian English' (IndE) is a term commonly applied to the variety(/ies) of English as used by speakers in India (and also by the Indian diaspora around the world). The vast majority of IndE speakers in India are native speakers of one or more indigenous Indian languages, and while some are exposed to English at home from a very early age, most are exposed from school (at varying stages). Despite the wide and generalized application of the term, the concept of IndE is somewhat hard to pin down, owing to a variety of factors, including vast linguistic diversity and complex multilingualism, factors themselves shaped by rapidly changing socio-economic conditions. The influence of indigenous L1s is strong [see 1,2,3] potentially leading to the identification of multiple varieties of IndE. However, these substrate features may themselves be convergent (identifiable as 'areal features', in segmental phonology [4] and prosody [5]), and other unified target features may also emerge in a process of standardisation of IndE [6].

There is thus on-going debate in the literature over the status of IndE [7,8,9] and the question of whether we are dealing with essentially a single pan-Indian variety or multiple sub-varieties $[6,7,10,11,12,13]$. While it has been established that IndE has some shared phonological features, irrespective of L1, more recent experimental research finds evidence for influence of specific L1s $[10,13,14]$, with the added complexity that the extent of L1 influence may also vary depending on the feature under investigation $[6,10,13]$. Any uniform approach to prosody, whether based on prosodic features of a native English variety or not, for example, is less likely to be taught explicitly, and so prosody may plausibly show greater L1 influence. The present paper adds to the body of research and examines the effect of L1 on the use of durational differences in IndE to signal, among other things, aspects of prosodic structure, in particular to mark lexical and sentential prominences and prosodic phrase boundaries.

In contrast to the fairly large number of studies on the segmental phonology of IndE, much less research has been conducted on its prosody in general, and in particular on aspects of duration and timing. Earlier studies on prosody have often been preliminary or based on a limited number of L1 backgrounds or/and speakers $[10,13,14,15]$. [6] examine Hindi and Telugu English and L1s. Work on rhythmic properties has claimed that IndE is more "syllable-timed" (a misnomer for expressing, essentially, lesser variability in vocalic and consonantal intervals), across various L1s and locations $[12,16]$ although as with rhythm research on other languages, results differ across metrics and speech style, and other parameters, such as $\mathrm{f0}$ and intensity are reported to be important [12]. There is evidence that IndE, depending on register and variety, may show certain temporal properties characteristic of native varieties of English, such as unstressed vowel reduction and phrase-final lengthening, but less than in e.g. British English [12]. [16], researching Telugu and Hindi English, find some evidence for L1 influence, but much more similarity than in the L1s themselves.

The purpose of this study is to investigate a set of temporal phenomena that are well-documented in native varieties of English, across a set of IndE varieties, as differentiated by the L1 of the speaker: Hindi, Bengali (Indo-Aryan), Telugu and Tamil (Dravidian). The four L1s in question are phonologically distinct, both segmentally and prosodically. All of them have a quantity contrast in consonants, but only Hindi, Telugu and Tamil have contrastive vowel length. Hindi has default prominence on the final syllable [17], while Telugu and Bengali have it on the first $[18,19]$. There is disagreement on the existence and location of stress in Tamil [20,21,22], but [23], reports that consistent differences in F0 give prominence to initial syllables. The phonetic signalling of stress in Indian languages is a point of debate, with $[17,24]$ claiming, on the one hand, that there is no phonetic cue to lexical stress in Hindi, and [25] claiming, on the other, that duration is a useful acoustic cue (together with pitch accent, see also [26]. Research suggests that vowel and syllable duration, together with pitch, are reliable markers of prominence also in Telugu [27]

[6] report greater phrase-final lengthening in Telugu than in Hindi, and evidence for this being replicated in the associated variety of IndE. They also report a differentiation in lexical stress patterning: for the production of the same set of disyllables, V2 was longer for Hindi speakers, and V1 longer for Telugu speakers. Rhythmically, Hindi has been described as 'syllabe-timed [28]; Telugu as 'mora-timed' [29] and Tamil variably as stress-timed [21,23,30]; syllable-timed [31], or neither [2], or mora-timed [32]. 
The temporal phenomena selected for investigation in this study were:

1) Phrase-final lengthening

2) The duration of tense and lax vowels

3) Stress-conditioned durational variation

If L1s exert a strong influence on varieties of IndE, we would predict that Bengali-English does not make durational distinctions between 'tense' and 'lax' vowels, given the lack of durational contrast in Bengali, but that the other varieties do. We also predict, since durational cues to lexical and phrasal prominences are, at best, subtle, in the L1s concerned, that durational cues to lexical and phrasal prominences in IndE would also be, at best, subtle. Finally, for phrase-final lengthening (PFL), given the lack of research on this in L1s, we are not in a position to make strong predictions. However, given that PFL is a widely attested phenomenon across many languages, and has been reported in both Telugu and Hindi, to differing degrees, we expect to find evidence across different varieties of IndE.

\section{Method}

\subsection{Speakers}

Six female and two male speakers of Indian English were recorded at the University of Hyderabad, India. All speakers were enrolled in a university degree at the time of data collection, had started learning English at the age of 4-7 years, identified as bi- or multilingual, and were aged 22 to 30 . Participants represented four L1 backgrounds ( 2 speakers each): Tamil, Telugu, Hindi and Bengali. Participants for each L1 spoke the same dialect, with the exception of Bengali. One L1 Bengali speaker (DS) grew up in Guwahati, Assam, and learned an Eastern variety of Bengali (she also lived in various places in India, and had been more exposed to British English in her education), while the speaker (SP) was from West Bengal and learned the Kolkata dialect.

\subsection{Materials and analysis}

The speakers were asked to read "The North Wind and the Sun" passage three times in a neutral voice, i.e. as if telling a story. The part of the story analysed consisted (across the two speakers per variety) of 87 IPs for Telugu-English, 79 IPs for TamilEnglish, 68 IPs for Bengali-English, and 77 IPs for HindiEnglish (differences across variety resulting from differences in prosodic phrasing in delivery, which may potentially be influenced by the size of accentual phrases in the L1 concerned).

The speakers were recorded in a quiet room using a Zoom $\mathrm{H} 4 \mathrm{nSP}$ audio recorder with an external lapel microphone. The recordings were made at a sampling rate of $44.1 \mathrm{kHz}$. For each speaker, the sound file deemed to have the most natural reading was selected for further analysis and converted into mono .wav files. The selected recordings were segmented and annotated using the WebMAUS services, Munich Automated Segmentation web platform [33]. The segmentation was manually corrected and annotated in Praat [34]. Additional Praat annotation included the following tiers: consonantal and vocalic intervals, syllable boundaries, degrees of prosodic prominence, intonational phrase boundaries (IP). Three degrees of prosodic prominence were identified for labelling: unstressed
(U), stressed (S) and nuclear stressed (SS). 'U' and 'S' were guided by dictionary-definitions of prominence pattern, although with the flexibility to adjust where the prominence pattern was clearly different in IndE, or if intonational phrasing placed prominences elsewhere. 'SS' was identified as being the most prominent syllable in the intonational phrase, as judged by the authors (one of whom is a native speaker of British English, and the other is a near-native speaker of Australian English). Syllables and segmental intervals at the beginning and end of IPs were also marked. Duration measurements were automatically extracted, using a script, for consonantal and vocalic intervals, and syllables.

\section{Results}

\subsection{Short vs long vowels}

Consistent with our predictions, Bengali-English speakers showed no distinction in duration between vowels that are phonetically long and short in British English, in either unstressed (Figure 1a) or stressed syllables (Figure 1b), with the one exception of one Bengali-English speaker making a distinction for the stressed condition. The general lack of distinction in Bengali-English is presumed to reflect the influence of Bengali L1, in which vowel duration is not phonologically contrastive, unlike the other L1s investigated. The exception, for one Bengali speaker and in the stressed context only, could be a result of a higher degree of exposure to British English during her education.

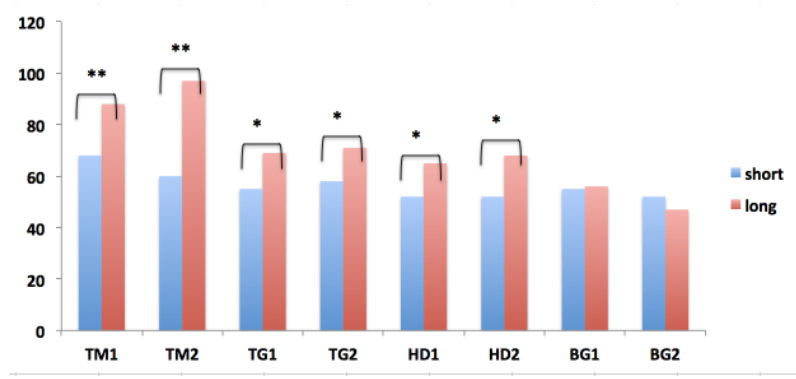

Figure 1a: Mean duration in ms of $V$ and $V$ : in unstressed syllables in phrase-medial position, across speakers and L1s

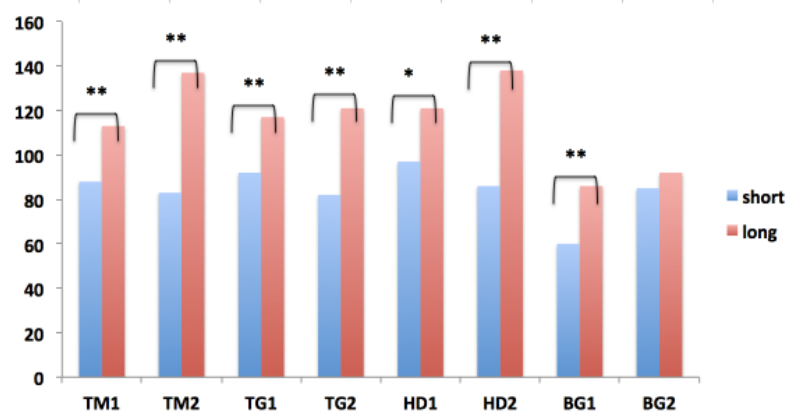

Figure 1a: Mean duration in ms of $V$ and $V$ : in stressed syllables in phrase-medial position, across speakers and L1s 
In all other varieties of IndE investigated, a distinction was made in both stressed and unstressed conditions. Again, this is consistent with our prediction that those speakers with an L1 with contrastive vowel length would be more likely to produce vowels of different durations in their IndE, even though the vowel duration in English is typically seen as one phonetic aspect of a more generalized quality contrast. Further, spectral, analysis is needed to examine whether these IndE speakers use durational distinctions as the only or most robust means to signal the vowel contrast.

The figures give results for medial position, where relevant data were most abundant, but this proved to be the case also for initial unstressed syllables and final nuclear accented syllables (for other conditions there were not sufficient data). For unstressed syllables, Tamil-English made the largest distinction, and in general, across varieties, the distinction was greater in the stressed condition.

\subsection{Stress-conditioned durational variation}

Contrary to our prediction, speakers of IndE from all four L1 backgrounds show systematic duration variation between stressed and unstressed syllables, in initial, medial (see Figure 2 ) and final position, with stressed syllables significantly longer than unstressed ones $(\mathrm{p}<0.001$ in all cases).

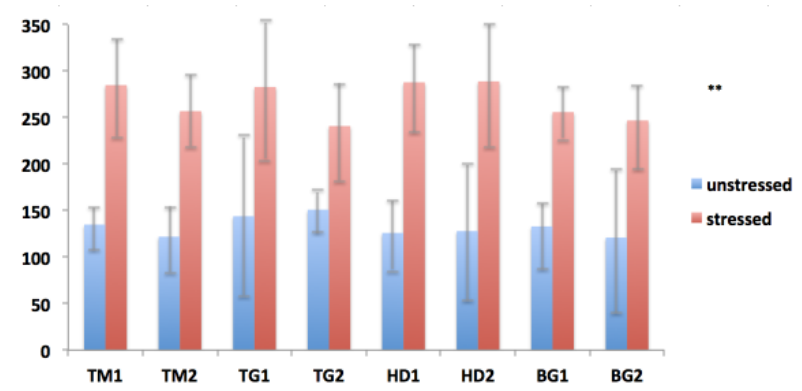

Figure 2: Mean duration in $m s$ of stressed and unstressed syllables, in phrase-medial position, across speakers and L1s

Looking at differences in the vowel duration alone, this pattern is replicated for Bengali- and Tamil-English in all phrase positions, while in Telugu- and Hindi-English it is only replicated in initial and medial position. Phrase-finally, vowels in stressed syllables are not significantly longer than those in unstressed syllables, suggesting that the systematic difference found at the level of the syllable phrase-finally is attained at least partially through consonant lengthening in the coda. Further investigation is required to determine this.

Additionally, while the distinction is statistically significant for both units measured, the distinction between stressed and unstressed syllables is notably greater than that between stressed and unstressed vowels in Tamil-English, suggesting that variation in consonant duration makes an important contribution also in this variety in the signalling of stress. Curiously, Bengali-English does not show this pattern suggestive of consonant lengthening, even though Bengali, uniquely among the L1s investigated, has contrastive length only in consonants and not in vowels. We conjecture that, even though there is no contrastive consonant length to signal in English, the specific constraints placed on consonant duration, and not on vocalic duration, in (Bengali) L1, may be exerting an influence here.

While there is a clear differentiation between stressed and unstressed syllables in terms of duration, none of the four IndE varieties examined show a significant distinction in the duration of stressed and nuclear-accented syllables. This raises the question as to whether the underlying L1s (and therefore the Indian Englishes on which they can be expected to exert an influence) have nuclear accents as understood and identifiable in e.g. British English varieties, and if they do, whether these are solely realized through pitch movement, and indeed whether there are different constraints on their precise realization in the phrase.

\subsection{Phrase-final lengthening}

The final source of durational variation investigated was the presence of lengthening at phrase-boundaries, specifically phrase-finally, since this is known to be a marked feature of British and American varieties of English. The results show different patterns for stressed and unstressed syllables.

For unstressed syllables, there is clear evidence of phrasefinal lengthening across all four varieties of IndE, particularly for Tamil-English and (to a lesser degree) in Hindi-English (see Figure $3 \mathrm{a}$, and also replicating findings by [6], for which the differences are highly statistically significant for both speakers. For Telugu-English and Bengali-English the trend is the same, but is highly significant in only one speaker of each.

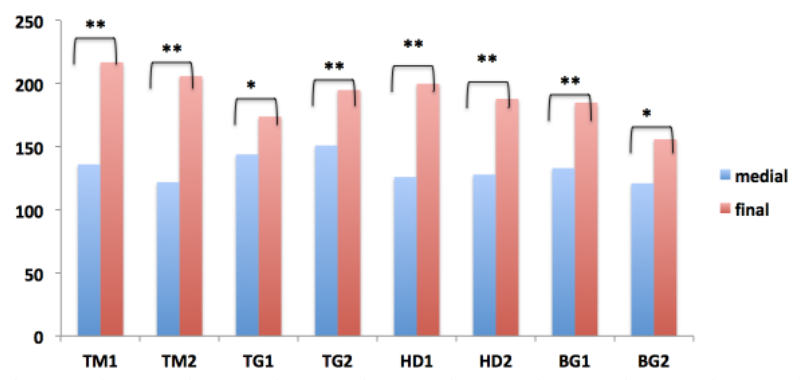

Figure 3a: Mean duration in ms of unstressed syllables in phrase-medial and phrase-final position, across speakers and L1s

For stressed syllables (see Figure $3 b$ ), while there is a trend for syllables to be longer phrase-finally, the difference is only statistically significant for one Bengali-English speaker. It has already been shown that all speakers lengthen the duration of prosodically prominent syllables (see section 3.2 ), and so we know that durations for this condition are already quite long (on average $>250 \mathrm{~ms}$ ), even when phrase-medial. While speakers show phrase-final lengthening in unstressed syllables (as we have also shown above), they appear not to mark stressconditioned lengthening and phrase-final lengthening cumulatively. It is conceivable that L1-mediated constraints are at play which limit the extent of durational variability between syllables, and thus the double-lengthening effect of prosodic prominence and phase-final position is effectively being suppressed. This would suggest that the general rhythmic properties of L1s (reported to be more evenly timed in all four 
L1s than in British English) may be playing a role in shaping how IndE speakers phonetically implement prosodic structure.

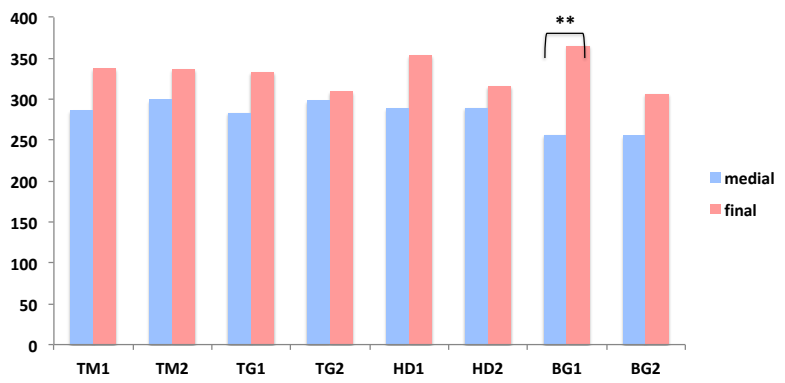

Figure 3b: Mean duration in ms of stressed syllables in phrase-medial and phrase-final position, across speakers and $L 1 s$

The divergent behaviour of the one Bengali-English speaker is interesting in this regard because this speaker reported having a higher degree of exposure to British English during her education. Her more generalized phrase-final lengthening may be due to greater acceptability to a change in rhythmic template as a result of this different exposure. Dialectal differences in her L1 Bengali may also play a role. More data, from a wider range of speakers, are needed in order to investigate this more fully.

\section{Discussion and conclusion}

This study set out to investigate the extent to which the IndE spoken by native speakers of different L1s shows uniformity in the production of a set of temporal properties, and the degree to which possible influence of L1 can be detected. The results present a somewhat complex picture, with some evidence of L1 influence (e.g. in the presence or absence of lexical vowel duration differences associated with tense and lax vowels), but also the pan-IndE adoption of temporal characteristics of native English varieties, such as phrase-final lengthening. The latter would appear to indicate that the target phonology and phonetic implementation of IndE are, at least in some respects, pan-Indic and distinct from native L1s spoken. Nevertheless, the extent to which this apparent adoption of a native English feature needs to be explored more carefully. Since there is little research on whether these L1s also display phrase-final lengthening, and if so to what extent, close phonetic analysis is needed of the same subjects speaking their respective L1s. The evidence of PFL may, in fact, be an example of convergence between a characteristic of native English varieties and Indian L1s.

A complete picture cannot be gained by looking at a temporal feature in isolation, either, since speakers may converge with the native target on one feature but vary on others. With regard to this, there is evidence of a possible panIndE 'resistance' to the cumulative application of structural 'triggers' to vowel lengthening, in that phrase-final lengthening is only evident in unstressed syllables. Stressed syllables, which are already longer through being prosodically prominent, do not lengthen significantly further as a result of being phrase-final. As discussed above, there may be more general, rhythmic properties of the L1 in question which shape how IndE speakers phonetically implement prosodic structure, and these might become more dominant in spontaneous speech, or in certain speech settings or styles.

A consequence of the lack of PFL in stressed syllables is that the durational cue to stress in these varieties of IndE is less acoustically salient. Since unstressed syllables do lengthen finally, the durational distinction with their stressed syllable competitors is decreased.

With regard to whether we are dealing with one or multiple varieties of IndE, it is difficult to determine from small dataset such as this how representative these results are for each of the IndE investigated, even for speech of the same register and by speakers of similar educational background and socioeconomic circumstances. Different kinds of speech elicitation and setting, including spontaneous speech, and speakers with varying degrees of education in English, would most likely introduce greater variation in timing between IndE of different L1s. The next steps in our research will involve, in addition to more speakers per variety, looking also at spontaneous speech recorded alongside the read passage. We shall also analyse associated temporal and prosodic phenomena in recordings made of their L1 speech to determine whether divergences can be attributed to the influence of a given L1, or to different levels of acquisition, or indeed to the existence of multiple forms of IndE, providing a choice of targets for the speaker. A closer examination of accent and phrasing is also needed, in close conjunction with analysis of L1s, in order to understand how durational cues are integrated with pitch movement in the signaling of prosodic structure.

\section{Acknowledgements}

We would like to thank the participants for taking part in this study, Professor Pingali Sailaja and the Centre for English Language Studies at the University of Hyderabad. The fieldwork was funded by the ARC Centre of Excellence for the Dynamics of Language through the Transdisciplinary and Innovation Grant (2016-2017). 


\section{References}

[1] R. K. Bansal, "A phonetic analysis of English spoken by a group of well-educated speakers from Uttar-Pradesh", CIEFL Bulletin (Hyderabad), vol. 8, pp. 1-11, 1970.

[2] T. Balasubramanian, "The vowels of Tamil and English: A study in contrast", CIEFL Bulletin (Hyderabad) vol, 9, pp. 27-34, 1972.

[3] Z. Thundy, "The origins of Indian English", CIEFL Bulletin (Hyderabad), vol.12, pp.29-40, 1976.

[4] C. Masica, Defining a linguistic area: South Asia. New Delhi: Chronicle Books, 2005.

[5] S. D. Khan, "The intonation of South Asian languages". FASAL6, Umass Amherst, 2016.

[6] H, Sirsa, and M.A. Redford, "The effects of native language on Indian English sounds and timing patterns". Journal of Phonetics, vol. 41(6), pp. 393-406, 2013.

[7] P. Sailaja, "Indian English: Features and sociolinguistic aspects." Language and Linguistics Compass, vol. 6(6), 359-370, 2012.

[8] J. Mukherjee, "Steady states in the evolution of new Englishes Present-day Indian English as an equilibrium", Journal of English Linguistics, vol. 35(2), 157-187, 2007.

[9] E. Schneider, Postcolonial English: Varieties around the World. Cambridge: CUP, 2007.

[10] C. Wiltshire, and J. Harnsberger, "The influence of Gujarati and Tamil L1s on Indian English: A preliminary study", World Englishes, vol. 25(1), pp. 91-104, 2006.

[11] R. Gargesh, "Indian English: Phonology", in E. Schneider, K. Burridge, B. Kortmann, R. Mesthrie, and C. Upton, Eds. A handbook of varieties of English: A multimedia reference tool (Vol. 1). Berlin, Germany: Mouton de Gruyter, 2004, pp. 9921002.

[12] R. Fuchs, Speech rhythm in varieties of English: Evidence from Educated Indian English and British English. Singapore: Springer, 2016.

[13] O. Maxwell, The intonational phonology of Indian English: An autosegmental-metrical analysis based on Bengali and Kannada English. Unpublished PhD dissertation, University of Melbourne, 2014.

[14] V. Puri, Intonation in Indian English and Hindi late and simultaneous bilinguals. Unpublished $\mathrm{PhD}$ dissertation, University of Illinois, 2013.

[15] R. Moon, A comparison of the acoustic correlates of focus in Indian English and American English. Unpublished Master's thesis, University of Florida, 2002.

[16] J. Krivokapić, "Rhythm and convergence between speakers of American and Indian English", Laboratory Phonology, vol. 4, pp. 39-65, 2013.

[17] M. Ohala, "Phonological areal features of some Indo-Aryan languages", Language Sciences, vol.13(2), pp. 107-124, 1991.

[18] Srinivas C. Word stress in Telugu and English: A study in contrast. M.Phil. dissertation, CIEFL, Hyderabad, India: 1992.

[19] P. Sailaja, Some Aspects of the Vowel Phonology of Telugu and Telugu-English. PhD thesis, CIEFL, 1985.

[20] M. S. Andropov, "Observations on accent in Tamil", International Journal of Dravidian Linguistics, vol. 2, pp. 111118, 1973.

[21] C. R. Marthandan, Phonetics of casual Tamil. Ph.D. dissertation, University of London, UK, 1983.

[22] N. S. Trubetzkoy, Principles of Phonology (translated by C. A. M. Baltaxe). Berkeley, CA: University of California Press, 1939/1969.

[23] E. Keane, "Prominence in Tamil", Journal of the International Phonetic Association, vol. 36(1), 1-20, 2006.

[24] M. Ohala, "A search for the phonetic correlates of Hindi stress. South Asian languages: structure, convergence, and diglossia", in Bh. Krishnamurti, C. Masica, and A. Sinha, Eds., 1986, pp. 81-92.

[25] R. Nair, A. Abbi, R. Gupta and A. Kidwai, "Acoustic correlates of lexical stress in Hindi", in Proc. Linguistic Structure and Language Dynamics in South Asia of the proceedings of SALA XVIII Roundtable, 2001.

[26] L. O. Dyrud, Hindi-Urdu: Stress Accent or Non-Stress Accent? Ph.D. dissertation, University of North Dakota, 2001.
[27] R. Balusu, "Acoustic correlates of stress and accent in Telugu", 21 st South Asian Languages Analysis Roundtable, University of Konstanz, pp. 7-19, 2001.

[28] R. M. Dauer, "Stress-timing and syllable-timing reanalysed", Journal of Phonetics. Vol. 11, pp. 51-69, 1983.

[29] L. Murty, T. Otake and A. Cutler, "Perceptual tests of rhythmic similarity: I. Mora rhythm". Lang. Speech, vol. 50, pp.77-99, 2007.

[30] E. Grabe, and E.L. Low, "Durational variability in speech and the rhythm class hypothesis", in C. Gussenhoven, and N. Warner Eds. Laboratory phonology: Vol. 7. Berlin: Mouton de Gruyter; 2002. pp. 515-546.

[31] G. Ravisankar, Intonation Patterns in Tamil. Pondicherry, India: Pondicherry Institute of Linguistics and Culture, 1994.

[32] F. Ramus, M. Nespor, and J. Mehler, "Correlates of linguistic rhythm in the speech signal”, Cognition, vol. 73, pp. 265-292, 1999.

[33] T. Kisler, U. Reichel, and F. Schiel, "Multilingual processing of speech via web services". Computer Speech \& Language, Vol. 45, pp. 326-347, 2017.

[34] P. Boersma, and D. Weenink, Praat: Doing phonetics by computer (Version 6.0.24), 1992-2017. Available from http://www.praat.org/ 